Revue d'histoire de l'Amérique française

REVUE D.HISTOIRE DE L'AMÉRIQUE FRANÇAISE

\title{
Présence et influence de Robert Mandrou au Québec
}

\section{François-Olivier Dorais}

Volume 69, numéro 3, hiver 2016

URI : https://id.erudit.org/iderudit/1035961ar

DOI : https://doi.org/10.7202/1035961ar

Aller au sommaire du numéro

\section{Éditeur(s)}

Institut d'histoire de l'Amérique française

ISSN

0035-2357 (imprimé)

1492-1383 (numérique)

Découvrir la revue

Citer cet article

Dorais, F.-O. (2016). Présence et influence de Robert Mandrou au Québec. Revue d'histoire de l'Amérique française, 69(3), 59-82.

https://doi.org/10.7202/1035961ar

\section{Résumé de l'article}

Cet article porte sur la relation privilégiée qui s'est établie, au cours des années 1960 et 1970, entre l'historien français Robert Mandrou et un groupe d'historiens québécois, jeunes pour la plupart ou en début de carrière. Il cherche à mettre au jour les conditions et la portée de ce rapprochement dont l'impact fut déterminant pour l'évolution de l'historiographie québécoise. L'auteur pose l'hypothèse que ce rapport évolue à travers la structuration d'un ensemble de « connecteurs intellectuels " qui se rapportent tant à la réception québécoise du paradigme des Annales qu'à la spécificité des réseaux de sociabilités que Mandrou a intégrés au Canada francophone. 


\title{
Présence et influence
}

\section{de Robert Mandrou au Québec ${ }^{\mathrm{I}}$}

\author{
François-Olivier Dorais \\ Département d'histoire \\ Université de Montréal
}

RÉSumÉ - Cet article porte sur la relation privilégiée qui s'est établie, au cours des années 1960 et 1970, entre l'historien français Robert Mandrou et un groupe d'historiens québécois, jeunes pour la plupart ou en début de carrière. II cherche à mettre au jour les conditions et la portée de ce rapprochement dont l'impact fut déterminant pour l'évolution de l'historiographie québécoise. L'auteur pose l'hypothèse que ce rapport évolue à travers la structuration d'un ensemble de «connecteurs intellectuels» qui se rapportent tant à la réception québécoise du paradigme des Annales qu'à la spécificité des réseaux de sociabilités que Mandrou a intégrés au Canada francophone.

ABstract • This article focuses on the special relationship established during the 1960s and 1970s between French historian Robert Mandrou and a group of, mostly, young historians in Quebec. It seeks to understand the conditions and the scope of this intellectual affinity which had a decisive impact on the evolution of Quebec historiography. The author posits that the relationship between Mandrou and these historians evolved through a series of "intellectual connectors" pertaining both to the French-Canadian reception of the Annales School and the sociability networks that the French historian constructed with Quebec.

1. Nos plus sincères remerciements à Jean-Philippe Warren, Daniel Poitras et aux deux évaluateurs anonymes de la revue pour leurs commentaires sur les versions antérieures de cet article. Merci aussi à Gérard Bouchard, Yvan Lamonde, Christine Piette, Martin Pâquet et André Larose pour leurs témoignages et précieuse collaboration. Nous tenons également à souligner l'appui financier du Conseil de recherches en sciences humaines du Canada (CRSH). 
D ans sa spécificité insulaire face aux grandes traditions scientifiques occidentales, la société québécoise a, comme dans la plupart des nations périphériques à l'Europe, souscrit aux idéologies d'emprunt dans la pratique de sa connaissance. De tout temps, des vecteurs scientifiques étrangers sont venus prendre part à la transformation de ses disciplines. L'étude de ces vecteurs d'emprunt - et tout particulièrement ceux venus de la France, qui s'imposent par leur régularité - est une condition primordiale en vue d'une juste appréciation de la configuration des savoirs d'ici. Si l'on a déjà maintes fois insisté sur l’importance des échanges historiographiques avec l'Hexagone dans le mouvement de disciplinarisation de la science historique ${ }^{2}$, très peu d'analyses ont, en revanche, cherché à interroger en profondeur la nature de ces échanges, leurs significations, leurs moutures idéologiques de même que les modes de réception auxquels ils ont donné lieu.

Pourtant, la rencontre des historiens québécois avec les historiens français durant les décennies d'après-guerre n'a rien d'un phénomène isolé et trouve peu de commune mesure dans les rapports entre le Québec et l'Angleterre ou les États-Unis. Elle s'effectue à la faveur d'un nouveau contexte socio-institutionnel suscité par la démocratisation et la massification de l'éducation universitaire, l'ouverture à l'international, mais aussi et surtout par l'intervention de l'État dans les secteurs de l'éducation et de la science, devenus les nouveaux opérateurs indispensables d'une «modernisation» de la société québécoise. Il convient de noter d’emblée que cette rencontre déploie une structure de sociabilité relativement complexe, étendue sur plusieurs années dans certains cas et à travers laquelle se nouent des liens de cordialité et de filiations durables. Ces liens donnent à voir des dynamiques de transfert, de solidarité, d'homologie et d'appropriation qui débouchent sur des productions concrètes sous forme de publications, de mentorat, de supervision, d'enseignement, de conférences ou de collaboration.

Au cour de ces circulations historiographiques entre les sphères universitaires française et canadienne-française se tient un historien, Robert

2. Sur ce point, on se référera notamment à Alfred Dubuc, "L'influence de l’École des Annales au Québec ", Revue d'histoire de l'Amérique française, 33, 3 (1979): 357-386; Ronald Rudin, Faire de l'histoire au Québec (Québec, Septentrion, 1998), 153-197; Pierre Savard, Entre France rêvée et France vécue. Douze regards sur les relations franco-canadiennes aux XIX et XX siècles (Montréal, Éditions Nota bene, 2009); Martin Pâquet et Patrick-Michel Noël, «L'Hexagone et la disciplinarisation de l'histoire au Québec: l'influence française sur l'historiographie québécoise, 1895-1963 ", Bulletin d'histoire politique, 20, 1 (automne 2011): 98-112. 
Mandrou, membre de la seconde génération du mouvement des Annales ${ }^{3}$. Rattaché à l'École pratique des Hautes Études et à l'Université de Nanterre, il figure parmi les quelques historiens français qui, avec André Latreille, Roland Mousnier et Alphonse Dupront notamment, seront invités dans le Québec d'après-guerre à prendre part au renouvellement de la science historique. Grand promoteur de l'histoire des mentalités et doté d'une sensibilité façonnée à la lecture de Michelet et sous l'influence déterminante de Lucien Febvre, dont il fut l'un des disciples les plus proches, Mandrou effectuera un total de sept séjours au Canada entre 1963 et $1975^{4}$, tous étant d'ordre strictement académique. Ses principales interventions auront lieu à l'Université Laval et à l'Université d'Ottawa, deux institutions avec lesquelles il tissa des liens serrés, ainsi que dans d'autres centres universitaires canadiens pour y prononcer diverses conférences et animer des séminaires de méthodologie historique et d'histoire européenne. Cette collaboration et cette influence se sont aussi prolongées jusqu'à Paris, où Mandrou prit sous son aile plusieurs étudiants durant leur formation supérieure en histoire au cours des années 1960 et 1970. Parmi ceux-ci, on recense quelques figures marquantes du paysage historiographique et intellectuel québécois contemporain, comme Fernand Ouellet, Gérard Bouchard, Louise Dechêne, André Sansfaçon, Régine Robin, Louise Beaudoin et Christine Piette.

De Mandrou, la postérité a retenu trop peu de choses, probablement en raison de son décès prématuré survenu en 1984, à l'âge de 63 ans, des suites d'une longue et pénible maladie. Il n'en demeure pas moins que grâce à l'établissement d'un réseau particulièrement durable avec le Québec, il fut à n'en point douter l'un des intermédiaires privilégiés de la "nouvelle histoire» française chez les générations d'historiens québécois d'après-guerre. L'étude de son parcours outre-Atlantique, facilitée par la découverte récente de son abondante correspondance avec le Canada ${ }^{5}$,

3. Cette seconde génération regroupe des figures aussi diversifiées que Fernand Braudel, Georges Duby, Pierre Goubert, Pierre Chaunu, Albert Soboul, Roland Mousnier et plusieurs autres. Pour une histoire détaillée du mouvement, voir André Burguière, L'École des Annales, une histoire intellectuelle (Paris, Odile Jacob, 2006).

4. Ces séjours académiques pouvaient parfois durer deux mois. Ils se sont déroulés au cours des années scolaires 1963, 1965, 1967, 1969, 1971, 1973 et 1975.

5. Cette correspondance a été recueillie dans le Fonds d'archives privé de Robert Mandrou aux Archives nationales de France, à Paris. Le corpus rassemble quelques centaines de lettres pour la plupart provenant de professeurs et d'étudiants de la région de Québec et d'Ottawa. Mandrou n'ayant malheureusement pas conservé toutes les copies carbones de ses propres lettres, il nous a été difficile de reconstituer la totalité des échanges, ce qui pose assurément une limite importante à notre recherche. Il n'en demeure pas moins que la quantité imposante de lettres reçues de la part de ses correspondants québécois, 
jette un nouvel éclairage sur sa présence et son influence au Québec, et tout particulièrement en ce qui a trait au développement de sa science historique.

Dans cet article, nous évoquerons l'itinéraire de Mandrou au Canada français en tâchant de mettre au jour et d'expliquer les liens de connivence et de complicité qu'il en est venu à nouer avec ce milieu. Dans l'intention d'élargir notre compréhension du rapprochement France-Québec à cette époque et surtout, de sa dynamique spécifique dans le domaine de l’historiographie, il nous est paru essentiel de rendre compte intellectuellement de la nature et de la signification de ce réseau universitaire. La rencontre entre un maître et ses disciples étant rarement le fruit du hasard, elle est aussi fonction de conditions sociales et idéologiques particulières qui modèlent les rapports de production, de transmission et de réception. Dans cet esprit, nous nous proposons de cerner la relative adéquation entre le parcours personnel et la sensibilité idéologique et disciplinaire de Mandrou, et les attentes, préoccupations et besoins particuliers qui traversent l'espace intellectuel et scientifique québécois des années 1960 . Nous posons l'hypothèse que ce rapport évolue à travers la structuration d'un ensemble de "connecteurs intellectuels» et de médiations qui se rapportent tant à la réception québécoise du paradigme des Annales qu’à la spécificité des réseaux de sociabilités que Mandrou a intégrés en ces lieux.

\section{LES CONDITIONS INTELLECTUELLES D'UN RAPPROCHEMENT}

En choisissant, au cours des années 1950, d'adapter la notion de mentalité au continent historique ${ }^{6}$, Mandrou faisait déjà, en quelque sorte, figure de "dissident» dans le champ historiographique français, à l'heure du triomphe de l'histoire économique et sociale. Il était d'ailleurs de ceux qui, en voulant rappeler l'importance des fondements culturels des différenciations sociales, avaient émis une mise en garde contre le réductionnisme économique des courants quantitatifs inspirés par les travaux du très influent Ernest Labrousse ${ }^{7}$. Au dire de Brigitte Mazon, cette dissidence aurait d'ailleurs servi de prétexte à Fernand Braudel pour écarter

parfois longues et très personnelles, attestent l'importance du réseau qu'avait tissé l'historien français de part et d'autre de l'Atlantique.

6. Bien que déjà évoquée sous la plume de Febvre et Bloch dans les années 1920 et 1930, l'expression «histoire des mentalités» reste surtout l'invention de Georges Duby et de Robert Mandrou, dans les années 1956-1960. Ce n'est toutefois qu'au cours des années 1970 que ce courant historiographique va véritablement connaître son apogée.

7. Voir notamment Robert Mandrou, «Mathématiques et histoire », Critica storica, 1, 1 (janvier 1962): 39-48. 
Mandrou du secrétariat de la revue des Annales en juin $1962^{8}$. Le geste trahissait toutefois une querelle bien plus profonde entourant l'héritage intellectuel de Febvre; l'homme d'institution et de pouvoir qu'était Braudel ne pouvait supporter que cet héritage ne lui revienne en totalité et surtout, qu'un jeune historien de solide concurrence ne donne au maître une postérité dans le champ des mentalités9.

Cet épisode vint ouvrir chez Mandrou une blessure dont la cicatrice ne se refermera jamais complètement ${ }^{10}$. Le sentiment de solitude et d'ostracisme qui devait résulter de cette épreuve l'incita sans doute à intégrer d'autres milieux historiographiques moins hostiles, à commencer par le Canada, dont il appréciait la "relative tranquillité québécoise ${ }^{11}$ ", mais aussi ses paysages et surtout, Québec, «la vieille ville», d'où il avait "découvert naguère le Nouveau Monde ${ }^{12}$ ». Il confiera d'ailleurs un jour à son collègue Marcel Trudel qu' "en attendant des temps meilleurs», il s' «efforc[erait] de rendre service aux étudiants de [1'Université] Laval sur tous les plans de son mieux $^{13}$ ». Sa familiarité avec la culture allemande conditionnera aussi le choix d'un exil plus tardif de l'autre côté du Rhin, à Göttingen, où il ira fonder en 1977 une importante mission historique française affiliée à l'Institut Max-Planck d'histoire.

Exclu du cercle braudélien, Mandrou n'en demeurait pas moins étroitement associé au paradigme annaliste qui, dans ses lignes de force, articulait un souci pour l'interdisciplinarité, la référence à une histoire-problème et la quête d'une troisième voie entre l'histoire événementielle dite «historisante», héritée des méthodistes français de la fin du XIX ${ }^{\mathrm{e}}$ siècle, et

8. Brigitte Mazon, «Introduction. Les archives de Robert Mandrou. "Une totalité vivante par la multiplicité ordonnée des plans et des abords" ", Les Cahiers du Centre de Recherches Historiques [En ligne], http:/ / ccrh.revues.org/2548 [consulté le 18 mai 2014].

9. Braudel accordait en effet peu d'importance aux «mentalités» dans sa conception de l'histoire, plutôt axée sur la «longue durée» et le poids du structurel, avec une forte connotation économiste. À l'inverse, l'histoire des mentalités privilégiait une utilisation plus marquée des temporalités historiques «courtes» et montrait une plus grande sensibilité pour l'événement et la rupture (sur la rupture personnelle et épistémologique entre Mandrou et Braudel, voir Jean Lecuir, "Robert Mandrou: Genèse de l'Introduction à la France moderne", dans Robert Mandrou, Introduction à la France moderne 1500-1640. Essai de psychologie historique [Paris, Albin Michel, 1998], 459-466).

10. Philippe Joutard et Jean Lecuir, «Robert Mandrou. L'itinéraire d'un historien européen du XX siècle», dans Histoire sociale, sensibilité collective et mentalité. Mélanges Robert Mandrou (Paris, Presses universitaires de France, 1985), 9-20.

11. Archives de l'Université d'Ottawa (AUO), Fonds Marcel Trudel (305), Mandrou, Robert: correspondance 1965-1969 - Lettre de Robert Mandrou à Marcel Trudel, 2 octobre 1965.

12. AUO, Fonds Marcel Trudel (305), Mandrou, Robert : correspondance 1965-1969, Lettre de Robert Mandrou à Marcel Trudel, 21 décembre 1966.

13. AUO, Fonds Marcel Trudel (305), Mandrou, Robert: correspondance 1965-1969, Lettre de Robert Mandrou à Marcel Trudel, 2 octobre 1965. 
les méthodes d'un marxisme plus orthodoxe ${ }^{14}$. Porté par l'aura de l'école française à l'échelle internationale mais aussi le succès d'un ouvrage initiateur sur les mentalités ${ }^{15}$, Mandrou fut invité pour un premier séjour en sol canadien à l'automne 1963, à l'occasion d'un important colloque international franco-québécois organisé par l'Institut d'Histoire de l'Université Laval. L'événement avait fait converger historiens, sociologues, politologues, démographes et linguistes du Québec et de la VIe Section de l'École pratique des Hautes Études. Trudel, alors directeur de l'Institut hôte, avait qualifié la rencontre d' «étape» et de "prise de conscience» dans l'évolution des relations intellectuelles avec la France par laquelle il s'agissait désormais d' «orienter [les] recherches selon des perspectives de convergence et de collaboration" de façon à marquer, espérait-il, un "effort décisif dans le dépassement de la période artisanale en histoire ${ }^{16}$.

L'orientation intellectuelle donnée au colloque, qui mettait à l'honneur diverses thématiques de l'histoire économique et de l'histoire des mentalités, préfigurait déjà l'influence profonde et diversifiée de l'école française au département d'histoire de Laval, qui en sera le principal foyer de réception au Québec. Il convient, à cet égard, de souligner combien ce département constituait, au-delà de la parenté linguistique, un lieu bien disposé pour recevoir les courants scientifiques français d'après-guerre. Dès sa fondation, en 1947, il avait moulé son enseignement sur le type européen, en s'inspirant du système des certificats de licence ${ }^{17}$. Plusieurs grands maîtres français, comme Pierre Deffontaines, Raoul Blanchard et André Latreille, y avaient également séjourné au cours des années 1940 et 1950 et au moins cinq de ses jeunes professeurs embauchés - Claude Galarneau, Fernand Ouellet, Jean Hamelin, Pierre Savard et Philippe Sylvain - avaient achevé leur formation en France.

Plusieurs jeunes historiens québécois, formés durant les années 1960, allaient trouver dans ce renouvellement des références à l'historiographie française l'horizon enviable d'une réforme intellectuelle et méthodologique de l'histoire. Un bref coup d'œil à la correspondance canadienne de Mandrou montre d'ailleurs combien ce dernier a pu marquer les esprits

14. François Dosse, L’histoire en miette: des Annales à la "nouvelle histoire» (Paris, La Découverte, 2010), 250.

15. Il s'agissait de son Introduction à la France moderne (1500-1640): Essai de psychologie historique, dont l'édition originale est parue en $1961 \mathrm{chez}$ Albin Michel.

16. Marcel Trudel, "Allocution d'ouverture», dans Claude Galarneau et Elzéar Lavoie, dir., France et Canada français du XVI ${ }^{e}$ au XXe siècle (Québec, Les Presses de l’Université Laval, 1966), 11-13.

17. Voir Marcel Trudel, «Les débuts de l'institut d'histoire à l'Université Laval», Revue d'histoire de l'Amérique française, 27, 3 (décembre 1973): 397-402. 
de tous ceux qui l'ont durablement fréquenté au fil de ses voyages. En 1965, Pierre Savard, alors jeune professeur à Laval, lui écrivait: «[...] l’enthousiasme des étudiants à la suite de votre séjour parmi nous témoigne de façon non équivoque que vous avez apporté beaucoup. Nous essaierons de ne pas laisser s'éteindre la flamme ${ }^{18}$. " Nombreuses sont aussi les lettres d'étudiants qui le remercient d'avoir provoqué chez eux un éveil et des orientations décisives. «C’est de vous que j’ai appris le plus, à Québec, à la fois le sens de l'histoire et la façon de pratiquer "le métier d'historien" ${ }^{19}$ ", lui écrivait en 1965 José Igartua, alors étudiant en lettres à l'Université Laval. René Hardy, étudiant en histoire et futur professeur à l'Université du Québec à Trois-Rivières, lui confiait pour sa part en 1968: «[...] le sens que vous avez donné à l'enseignement et à la recherche en histoire resteront pour toujours les lignes directrices d'une carrière que je désire aussi exemplaire que la vôtre ${ }^{20}$ ».

Ces témoignages d'admiration et de gratitude, dont nous pourrions multiplier les exemples, rendent bien compte de la séduction et de l'influence intellectuelle que pouvait exercer Mandrou auprès de la relève historienne québécoise. En entrevue avec François Ricard en 1981, l'historienne Louise Dechêne, dont Mandrou avait dirigé sa thèse de doctorat qui devait paraître sous le titre de Habitants et marchands de Montréal au XVII siècle, parle pour sa part d'une "influence indirecte». Plus proche du modèle braudélien par découpage structural ${ }^{21}$, Dechêne mesurait l'apport de son directeur surtout du point de vue de l'encadrement à valeur initiatique qu' il lui avait offert: "ce que j'ai fait ne ressemble en rien aux travaux de Mandrou, mais c'est quand même quelqu'un qui me faisait découvrir un tout en histoire à ce moment-là ${ }^{22}$ ».

Dès lors, pour comprendre les ressorts de cette complicité intellectuelle entre les étudiants québécois et l'historien français, il faut d'abord voir en quoi elle se lie à la force de novation qu'incarnait le courant des Annales dans le Québec de la Révolution tranquille. Dans ses formulations originelles, les Annales proposaient d'engager une véritable «repolitisation de

18. Archives nationales de France (ANF), Fonds Robert Mandrou (AB XIX), Correspondance générale (4475), dossier «S» - Lettre de Pierre Savard à Robert Mandrou, 4 décembre 1965.

19. ANF, Fonds Robert Mandrou (AB XIX), Correspondance générale (4475), dossier «H» - Lettre de José Igartua à Robert Mandrou, 23 septembre 1966.

20. ANF, Fonds Robert Mandrou (AB XIX), Correspondance générale (4475), dossier «H» - Lettre de René Hardy à Robert Mandrou, 12 juin 1968.

21. R. Rudin, Faire de l'histoire au Québec, op. cit., 207.

22. Louise Dechêne, "Écrire l’histoire au Québec, 1981 », dans Éric Bédard et Julien Goyette, dir., Paroles d'historiens. Anthologie des réflexions sur l'histoire au Québec (Montréal, Presses de l'Université de Montréal, 2006), 233. (Les italiques sont de nous) 
la science " par la diffusion d'un contenu à double visée programmatique : celle d'un «désasservissement idéologique de l'histoire» et la réaffirmation de son ambition scientifique ${ }^{23}$. Ainsi, à la suite de Daniel Poitras, nous estimons que les enjeux de la réception québécoise des Annales résident moins dans l'adoption de ses méthodes et de ses outils d'analyse que dans sa capacité à imprimer «le sens d'une mission scientifique», solidaire d'une intention épistémologique et critique visant précisément à rompre avec "l'histoire traditionnelle»" Sous cet éclairage, Mandrou pouvait apparaître comme une formidable caution scientifique et épistémologique pour les nouvelles générations d'historiens québécois d'après-guerre, caution par laquelle il devenait possible de reformuler - et, par la même occasion, disqualifier - la manière ancienne, événementielle et apologétique, de pratiquer l'histoire.

La légitimité de la référence scientifique, incarnée par Mandrou et les Annales, doit donc être considérée parallèlement à la conscience de rupture d'avec le Québec traditionnel qui s'annonce et se précise chez l'intelligentsia de l'époque et au besoin conséquent d'une nouvelle lecture cohérente du réel. Elle a partie liée au développement d'une historiographie critique de la mémoire canadienne-française et soucieuse de donner sens à une nouvelle modernité québécoise. Ce besoin d'un nouveau cadre de référence historique, en rupture de ban avec les injonctions théologiques et morales du passé, est exemplifié par l'orientation des enseignements que vient livrer Mandrou au Canada ${ }^{25}$.

Il n'est que de se tourner vers le corpus de ses notes de séminaires et de conférences donnés au Québec pour voir combien son propos tablait entre autres sur la distinction fondamentale à établir entre ce qui relève de l'attitude scientifique et de la mémoire collective. Dans l'un de ses plans de cours pour le Canada, on y retrouve par exemple une définition de la science comme une «mise à distance face à la mémoire socialisée et

23. Bertrand Müller, " "Histoire traditionnelle" et "histoire nouvelle" : un bilan de combat de Lucien Febvre», Genèses, 34 (1999): 133.

24. Daniel Poitras, «L'impossible oubli: Fernand Ouellet, la Révolution tranquille et la république contrefactuelle des patriotes", Revue d'histoire de l'Amérique française, 66, 3-4 (hiver-printemps 2013), 350. (Les italiques sont dans le texte original).

25. Le fonds Mandrou fait notamment état de ses voyages effectués à l'automne 1969 et à l'automne 1971, au cours desquels il mit sur pied à Laval et à Ottawa quelques séminaires portant sur diverses thématiques en histoire européenne : l'histoire de la sorcellerie, la médecine et les possessions diaboliques, les intellectuels et la vie intellectuelle en France au XVI' siècle, l'Ancien Régime et la Révolution française, le jansénisme français au XVII ${ }^{\mathrm{e}}$ siècle. Le fonds témoigne aussi de la dizaine de conférences qu'il prononça au Québec sur la méthode historique, le métier d’historien, les événements de Mai 68, la Révolution française et les divers aspects de l'histoire des mentalités en France. 
constituée de façon commémorative», attitude qu'il s'agissait d' "assumer, même si l'image de Jeanne d'Arc ou de Napoléon doit en souffrir». La «responsabilité sociale» de l'historien consistait dès lors à "agir» sur la mémoire collective en "confront[ant] mythe et réalité», peu importe "l'intérêt des mythes pour le personnel politique ou les groupes privés». Autrement dit, l'historien devait apprendre à «refuser tout embrigadement qui serait simple ralliement à des positions politiques, idéologiques, simplistes en fonction de quelques [...] catégories [...] ici libéral et conservateur, fédéraliste et nationaliste $»^{26}$.

La rupture avec une conception subjective et purement passéiste du discours historique n'impliquait pas, selon Mandrou, que l'histoire renonce à devenir un moyen fécond pour réfléchir sur le présent. L'histoire, selon la bonne vieille antienne annaliste, devait au contraire s'établir comme une "science du présent» et entretenir un rapport étroit avec l'actualité, laquelle était «toujours appelée au secours du démonstrateur ${ }^{27}$ ». Ce rôle s'envisageait alors moins sous l'angle d'une glorification du passé national et d'une actualisation de son intentionnalité que d'une capacité à interroger le passé de manière à jauger réalistement les problèmes et les inquiétudes de la société $^{28}$. L'enseignement de Mandrou pouvait donc, par un mouvement naturel, faire son lit d'une Révolution tranquille qui en confirmait implicitement les prémisses. C'est Jean Hamelin qui a probablement le mieux décrit en quoi les Annales françaises se sont faites solidaires du rôle proprement civique que plusieurs souhaitaient alors insuffler à la connaissance historique dans le Québec des années 1960:

Quand les traditions se désagrègent et les lieux de la mémoire collective disparaissent, on recourt à l'histoire pour raccorder le présent au passé. L'histoire que pratiquent les Annales d'alors convient parfaitement au temps de la Révolution tranquille. Elle n'est ni trop conceptuelle ni trop hermétique, au point d'être en rupture de ban avec le public; problématisée sans être dogmatique, elle accorde assez de place à la liberté pour valoriser les combats et les choix quotidiens; elle est suffisamment quantifiée pour donner de la profondeur à l'événement et stimuler les praticiens des sciences sociales ${ }^{29}$.

26. ANF, Fonds Robert Mandrou (AB XIX), dossier «Méthodologie» - Cours sur le métier d’historien et d'enseignant en histoire».

27. Ibid.

28. Jean Lamarre, Le devenir de la nation québécoise selon Maurice Séguin, Guy Frégault et Michel Brunet (1944-1969) (Québec, Septentrion, 1993), 395.

29. Jean Hamelin, «L'histoire des historiens : entre la reconstruction d'une mémoire collective et la recherche d’une identité. 1992», dans É. Bédard et J. Goyette, dir., op. cit., 214. 
On est frappé par les convergences entre ce commentaire d'Hamelin et une conférence que prononçait Mandrou à l'Université Laval le 2 octobre 1969 sur l'importance de la Révolution française dans l'histoire du Québec. Si nous ne disposons pas du texte original de l'allocution, le compte rendu effectué par un étudiant, André Larose, en restitue la matière essentielle. S'interrogeant sur l'absence de tradition révolutionnaire dans la mémoire collective québécoise, Mandrou constatait qu’à la différence de la France, il y avait eu, au Canada français, «sublimation de l'Ancien Régime» après la Conquête de 1763 et refoulement de la France révolutionnaire, républicaine et "progressiste» par les prêtres réfractaires venus d'Europe. Cette attitude avait, selon lui, entraîné une "grande mythification du destin québécois", un constat douloureux qui devait interpeller l'historien dans son travail d'élucidation du passé. Et l'étudiant Larose de conclure, en s'inspirant manifestement des conclusions de l'historien français: «Nous ne comblerons pas seulement les lacunes de notre historiographie ainsi. Plus encore: nous expliquerons au peuple québécois ce qu'il est; nous essaierons de le libérer de lui-même. Ce sera la part des historiens dans la libération du Québec ${ }^{30}$ ». De tels constats mettent en pleine lumière certains paramètres idéologiques fondateurs de la Révolution tranquille parmi lesquels il faut noter la rupture avec les consensus culturels du nationalisme canadien-français et la disqualification du traditionalisme.

Ainsi, il est aisé de voir combien les méthodes, les techniques et les interprétations importées par Mandrou pouvaient convenir parfaitement à de jeunes historiens désireux de marquer une rupture avec leurs prédécesseurs et déterminés à décrire les Québécois comme un peuple nordaméricain normal, moderne et libéré de toute référence à la société traditionnelle européenne. Le sociologue Joseph Yvon Thériault rappelle, à ce propos, combien la tradition de l'histoire sociale des Annales, en tablant sur une lecture critique et objective de la condition québécoise, s'était fait, aux côtés des études marxistes et de la sociologie quantitative américaine, l'agent promoteur d'une pratique historienne "explicative» plutôt que "compréhensive». Autrement dit, à une pratique qui prendrait d'abord en considération le caractère subjectif et singulier de l'expérience historique québécoise, les Annales auraient substitué le projet d’une science

30. André Larose, "La révolution française vue du Québec», La bombe H. Journal des étudiants en histoire, 7 novembre 1969 , p. 6-7. 
davantage axée sur l'exposition de ses régularités rationnelles et de son développement normalisé en contexte nord-américain ${ }^{31}$.

Sur un autre plan, le recours aux Annales dans le Québec des années 1960 se voulait aussi une réponse à l'omniprésence qu'avait acquise la sociologie américaine depuis la guerre ${ }^{32}$. Le parcours scolaire de Gérard Bouchard atteste ce phénomène, lui qui avait choisi de quitter la chapelle des sociologues de Laval pour intégrer, au $3^{\mathrm{e}}$ cycle de sa formation, celle des historiens. Cette conversion, Bouchard l'attribue en partie à la prégnance à l'époque d'une certaine tradition scientiste de la sociologie américaine et de sa vision "très mécaniciste [à la] recherche des modèles, des lois du changement social ${ }^{33}$ ». De ce point de vue, sa rencontre personnelle avec Mandrou, qui assurera la direction et la publication de sa thèse doctorale à Paris, fut capitale; au contact des Annales, Bouchard retrouvait une sensibilité recherchée pour la «durée» et «l'imprévu du mouvement social ${ }^{34}$ ». Il trouvait aussi, chez Mandrou, la figure d'un maître, dont la compétence n'avait d'égal que l'autorité naturelle:

C'était un homme qui avait des exigences intellectuelles et scientifiques extrêmement élevées, une érudition considérable, une rigueur redoutable. [...] c'était vraiment l'image du maître, qui était d'ailleurs encore très présente dans la culture de l'université française de l'époque. Il était, pour ainsi dire, comme une sorte de père ${ }^{35}$.

Derrière cette proximité intellectuelle, on soupçonne aussi une proximité de classe. Lui-même fils de cheminot parisien, et boursier durant ses études secondaires à Saint-Étienne, Mandrou ne cachait pas sa sensibilité et sa sympathie pour les étudiants issus de milieux modestes qui souhaitaient paver leur voie dans le monde universitaire. Bouchard, tout comme Fernand Ouellet d'ailleurs, avec qui Mandrou nouera des liens très étroits, semble confirmer cette règle; tous deux étaient représentatifs de cette première génération de diplômés québécois qui, issus de milieux ruraux et ouvriers, avaient bénéficié du mouvement d'expansion universitaire des années 1960.

31. Joseph Yvon Thériault, Critique de l'Américanité. Mémoire et démocratie au Québec (Montréal, Québec Amérique, 2005), 190-200.

32. A. Dubuc, «L'influence de l’École des Annales au Québec», loc. cit., 370.

33. François Baby, «Gérard Bouchard, Prix Léon-Gérin 1993 », Saguenayensia, 36, 2 (avril-juin 1994): 23.

34. Id. La thèse de doctorat de Bouchard, intitulée Le Village Immobile - Sennely en Sologne au XVIII siècle, paraîtra en 1971 chez Plon, dans la collection "Civilisations et mentalités », codirigée par Mandrou et Philippe Ariès.

35. Gérard Bouchard, entrevue, Montréal, février 2014. 
Il faut souligner un dernier aspect qui éclaire les confluences actives entre Mandrou et les historiens québécois. Celui-ci concerne l'élargissement de la problématique historienne aux questions sociales, tendance qui doit être corrélée avec la situation de déséquilibre provoquée par les effets conjugués d'une industrialisation, d'une démographie et d'une urbanisation croissantes. Les espoirs et les inquiétudes suscitées par ces changements ont mis en évidence un nouvel axiome de la pensée québécoise : celui d'une exigence nouvelle de totalité qu'Yves Couture associait au "globalisme». Cet imaginaire global conduit alors «à lier nécessairement les uns aux autres tous les problèmes de la nation, dont la cause commune est sa situation défavorisée, et à identifier également une solution commune qui passe par la transformation de cette situation globale au moyen de l'action de l'État ${ }^{36} »$. Il procède aussi du réaménagement de la perspective nationale canadienne-française sur la base d'une nouvelle territorialité - celle du Québec - qui, en quête d'une nouvelle plénitude identitaire, appelait la délimitation d'un nouvel espace politique, économique et social. Il est dès lors aisé de constater comment l'histoire sociale, en proposant une affiche englobante à la mesure des ambitions unificatrices des Annales, pouvait entrer directement en adéquation avec ces nouvelles représentations.

Accompagnant ce mouvement, Mandrou fit une contribution importante dans la première livraison de la revue Histoire sociale/Social history en 1968, une revue bilingue fondée à Ottawa par le professeur Marcel Trudel et quelques collègues et consacrée exclusivement à l'histoire sociale. Pour l'occasion, Trudel invita Mandrou à signer l'«article-programme» de la revue, requête que l'historien français accueillit en témoignage "d'une fidélité dans l'amitié», d'autant plus que l'interpellé avouait «rêver [...] de retrouver une tribune [perdue aux Annales], dans de bonnes conditions $^{37}$ ». La relation étroite qui unit Mandrou aux historiens québécois s'éclaire à travers cette collaboration, où convergent des engagements d'une même nature envers le projet d'une histoire sociale à établir. "L'histoire sociale est mère de toute histoire », écrivait-il dans la première livraison de la revue. "Tant de retards aussi, s'expliquent précisément parce qu'elle se retrouve présente dans toute tentative de reconstitution historique, animée d'une ambition élevée et soucieuse de rigueur scien-

36. Yves Couture, La Terre promise. L'absolu politique dans le nationalisme québécois (Montréal, Liber, 1994), 72.

37. AUO, Fonds Marcel Trudel (305), Mandrou, Robert: correspondance 1965-1969 - Lettre de Robert Mandrou à Marcel Trudel, 21 décembre 1966. 
tifique. Ce qui rend son avancement d'autant plus urgent et nécessaire: la faire progresser est certainement aujourd'hui le premier devoir des historiens». Un tel projet de connaissance impliquait, pour Mandrou, la prise en compte des dimensions plus larges d'un récit qui tiendrait désormais compte de «l'avènement du collectif » dans la problématique historique, c'est-à-dire en prenant pour objet ce qui relève d'abord des "peuples, sinon [des] civilisations de l'humanité tout entière $»^{38}$.

\section{COMPLICITÉS ET RÉSEAUX DE SOCIABILITÉS}

\section{Mandrou et l'uécole historique de Laval »}

Pour l'historien François Dosse, les réseaux et les milieux de sociabilités sont des «connecteurs» essentiels en histoire intellectuelle, en cela qu'ils permettent d'isoler les «micro-climats singuliers» qui structurent la vie des idées ${ }^{39}$. De ce point de vue, il n'est pas inutile de faire remarquer que Mandrou a surtout tissé des liens avec les milieux historiographiques de Québec et d'Ottawa ${ }^{40}$. Cela s'explique en partie, nous semble-t-il, par la solidarité de pensée qui l'unissait aux historiens de ces deux institutions, intellectuellement plus proches des thèses de «l'école historique de Laval $^{41}{ }^{\prime}$. Cette solidarité outrepassait les enjeux relatifs à l'imprégnation des Annales précédemment discutés; elle relevait aussi de choix épistémologique et politique évidents.

Déjà, en 1965, à l'occasion d'un colloque étudiant organisé à Laval, Mandrou émettait quelques réserves à propos de la thèse défendue par Michel Brunet sur la «déchéance» de la bourgeoisie d'affaire en NouvelleFrance à la suite de la Conquête de 1760 . Reprenant les conclusions déjà formulées par Fernand Ouellet et Jean Hamelin quelques années auparavant $^{42}$, Mandrou entrevoyait difficilement à son tour comment un modèle de bourgeoisie similaire à celui de la France métropolitaine d'Ancien Régime avait pu prendre forme en Nouvelle-France. Consciente de ses objectifs communs, cette bourgeoisie française des XVII ${ }^{e}$ et XVIII ${ }^{e}$ siècles

38. Robert Mandrou, "Primat de l'histoire sociale: propos sans paradoxes", Histoire sociale/Social History, 1 (avril 1968): 7-15.

39. Voir François Dosse, «L'irréduction dans l'histoire intellectuelle», Espace Temps, 84, 1 (2004): 172-186.

40. Encore ici, la correspondance reçue de Mandrou est un indicateur précieux de son réseau de sociabilités québécois. De fait, aucune lettre ne semble avoir été échangée avec des représentants des milieux intellectuels et universitaires montréalais. Celles-ci proviennent exclusivement des universités Laval et d'Ottawa.

41. Sur l'école de Laval en histoire, on se référera à R. Rudin, op. cit., 153-197.

42. Voir Fernand Ouellet, " M. Michel Brunet et le problème de la Conquête ", Bulletin des recherches historiques, 62, 2, (avril-juin 1956), 92-101; Jean Hamelin, Économie et société en Nouvelle-France (Québec, Les Presses de l’Université Laval, 1960). 
ne pouvait atteindre, selon lui, une forte conscience de classe du fait de son manque d'homogénéité et de son écartèlement entre ses diverses évasions sociales. Par ailleurs, pour peu qu'il y ait eu, en Nouvelle-France, "quantités de petits nobles à la recherche de meilleurs fiefs qu'en France [...] les conditions d'existence, les possibilités climatiques et géologiques n'[étaient] pas, selon Mandrou, favorables à une transplantation pure et simple des habitudes de travail et d'esprit communes en France ${ }^{43}$ ».

Cette mise en cause par Mandrou de l'une des thèses centrales de l'école historique néonationaliste préfigurait une critique encore plus vive des historiens de l'Université de Montréal, critique dont il donnera la pleine mesure dans un texte paru quelques années plus tard dans les pages de la Canadian Historical Review: "les historiens attendent toujours, patiemment, les œuvres scientifiquement élaborées que l'“école de Montréal”, plus attachée aux vertus oratoires qu’à la démonstration imprimée, doit nous donner un jour pour fonder en méthode et raison ses visions apocalyptiques du devenir canadien ${ }^{44} »$. Par ces propos, Mandrou s'alignait, et nourrissait en retour, une tendance touchant alors l'ensemble de la profession historienne et qui consistait à déprécier l'approche politique et la question nationale. De ce point de vue, il n'est pas surprenant de constater que l'historien français se sentait intellectuellement plus proche des historiens lavallois, dont le programme, élaboré initialement à Québec et poursuivi ensuite à l'Université d'Ottawa ${ }^{45}$, privilégiait une approche plus sociale et économique de l’histoire. Pour Mandrou, «Fernand Ouellet, comme Jean Hamelin, a[vaient] hérité de [leur] premier maître, Marcel Trudel, le sens du devenir de la recherche, le goût du progrès scientifique constamment nourri par le recours aux sources, par le retour - entre deux périodes d'enseignement - aux archives ${ }^{46}$ ».

Des trois historiens, Trudel était probablement celui avec qui Mandrou, par-delà les liens de cordialité qui les unissaient, se sentait le moins d'affinité épistémologique. Sans rien enlever à la grandeur et à l'importance de l'œuvre, l'historien français considérait toutefois que la méthode aux allures de «chroniques» privilégiée par le spécialiste du régime français

43. José Igartua, "Conférence de Robert Mandrou. Bourgeois français, Bourgeois canadiens", Le Carabin, 19 novembre 1965, 5.

44. Robert Mandrou, "L'historiographie canadienne française. Bilan et perspective», Canadian Historical Review, 51, 1 (mars 1970): 5.

45. Grâce notamment à l'installation au département d'histoire de l'Université d'Ottawa de nouveaux professeurs comme Marcel Trudel, Serge Gagnon, Louise Dechêne, Marcel Hamelin (futur recteur de l'institution), Pierre Savard et Fernand Ouellet, tous formés à l'Université Laval.

46. R. Mandrou, «L'historiographie canadienne française... », loc. cit., 18. 
comportait d'importantes limites: «Assurément, cette histoire-chronique ne peut apporter au lecteur de perspective vraiment neuve sur le devenir de la Nouvelle-France : tout au plus une illustration des thèmes plus classiques dont l'histoire de la colonisation est nourrie depuis que les entreprises françaises ont été passées au crible ${ }^{47}$. "En outre, il écrira à propos de L'Église canadienne sous le régime militaire, un ouvrage publié par Trudel à la fin des années 1950, que "le résultat n'est pas très heureux parce que l'auteur a voulu retracer le destin de chaque institution (dans son second volume) l'une après l'autre, sans souci des perspectives d'ensemble auxquelles G. Frégault est toujours attentif ${ }^{48}$ ».

Il semble que ce soit plutôt avec Fernand Ouellet, l'historien le plus polémiste du groupe lavallois mais acquis aux méthodes de l'histoire sociale, que les liens de proximité personnels et intellectuels furent les plus étroits. Mandrou ne tarira pas d'éloges à l'égard de l'Histoire économique et sociale du Québec, ouvrage paru en 1966 et qu'il qualifiera de "plus beau fleuron de la production de l'Institut d'Histoire de l'Université Laval, voire le plus important des livres d'histoire publiés au Canada depuis la fin de la Seconde Guerre mondiale ${ }^{49}$ ». Rappelons qu'il en avait aussi été le préfacier, autre signe évocateur d'une confluence intellectuelle particulièrement forte entre les deux hommes. Si Ouellet avait eu le mérite d' "esquiss[er] une histoire totale du Québec», ses recherches novatrices explicitaient surtout, selon Mandrou, «une vision fort originale [du] passé, si souvent invoquée de façon simpliste, en ces années difficiles, par les différents nationalismes québécois " ${ }^{50}$.

Cette préface en avait manifestement convaincu plus d'un sur l'existence d'une solidarité épistémologique bien établie à Laval. C'est le cas notamment de l'étudiant Jacques Mathieu, qui écrivait à Mandrou en octobre 1966:

La préface de l'ouvrage de F. Ouellet témoigne d'une foi convaincue en une certaine école de pensée, en une certaine forme de recherche naissante au Canada français. L'hommage que vous rendez à Ouellet rejaillit sur ceux qui ont failli être de ses étudiants. Enfin, peut-être serons-nous de ses disciples, tout au moins des disciples de l'équipe dont il est un des membres moteurs ${ }^{51}$.

47. Ibid., p. 9.

48. Ibid., p. 12 .

49. Ibid., p. 17.

50. Robert Mandrou, «Préface», dans Fernand Ouellet, Histoire économique et sociale du Québec 17601850 (Montréal, Fides, 1966), VII.

51. ANF, Fonds Robert Mandrou (AB XIX), Correspondance générale (4475), dossier «M» - Lettre de Jacques Mathieu à Robert Mandrou, 20 octobre 1966. 
Il y aurait aussi long à dire sur l'abondante correspondance échangée entre Mandrou et Ouellet, qui témoigne notamment de l'important travail de mentorat que le premier offrit au second dans la préparation de son manuscrit, notamment en ce qui concerne l'orientation de la longue conclusion sur la résistance des «mentalités d'Ancien Régime». L'historien français répondait aux envois de Ouellet par des commentaires, des appréciations, des points d'interrogation variés, des corrections stylistiques, des demandes d'éclaircissements et des conseils sur la production. Ainsi, la correspondance entre les deux historiens devenait le support d'une forme de critique scientifique, si ce n'est celle qui caractérise la relation privilégiée entre un maître et son élève.

Le penchant favorable de Mandrou, et vice-versa, pour la petite communauté historienne de Laval et, plus particulièrement, l'historiographie de Ouellet, trouve une explication convaincante dans le terreau critique commun face à l'optique nationaliste en histoire. Cette tendance se donnait à être tout particulièrement renforcie dans le champ de l'histoire socioculturelle: «Le processus de sensibilisation de l'historien au culturel s'est opéré d'abord parmi les historiens qui avaient pris leur distance par rapport au nationalisme (c'est aussi le cas des sociologues) et s'inscrit dans la logique du développement progressif de l'historiographie autour du concept d'histoire globale ${ }^{52}$ ", écrivait Ouellet en 1975. Comme plusieurs intellectuels français de sa génération, et en particulier ceux issus de la filière des Annales, Mandrou était un homme de gauche, d'un marxisme tempéré mais jamais tenté par le communisme, laïque et de sensibilité socialiste, dont l'engagement scientifique s'était précisé dans l'«esprit de la Libération» de l'aprèsguerre et son effervescence théorique longtemps dissimulée sous le dogmatisme et le pessimisme des idéologies totalitaires ${ }^{53}$.

Difficile aussi de ne pas relier la sensibilité épistémologique de l'historien avec l'expérience tragique qu'il avait personnellement vécue au début des années 1940, période durant laquelle il fut contraint à l'abandon des études pour rejoindre les chantiers de jeunesse et, de là, le service du travail obligatoire en Allemagne, où il fut envoyé comme bûcheron dans la forêt du $\mathrm{Harz}^{54}$. On soupçonne facilement combien cette expérience avait pu nourrir chez lui la perception doctrinaire et dangereuse des sentiments nationalistes. À tout le moins, l'approche nationale en histoire lui

52. Fernand Ouellet, "Colloque d'histoire socio-culturelle. Symposium in Socio-cultural History. L'histoire socio-culturelle: colloque exploratoire », Histoire sociale/Social history, 9, 17 (mai 1976): 6.

53. A. Burguière, L'École des Annales..., op. cit., 19.

54. P. Joutard et J. Lecuir, «Robert Mandrou. L'itinéraire... », loc. cit., 9-10. 
inspirait-elle la méfiance en cela qu'elle naturalisait des différences attribuables aux situations sociales:

[...] les différences à mon sens l'emportent sur les traits communs. Ce qui n'est pas facile, ni agréable peut-être, à reconnaître: nous vivons sur ce plan nourri de mythes, d'idées fausses qui ont fait leur chemin à travers les idées reçues lénifiantes de la para-histoire, des communications de masse, voire à travers certaines formes d'enseignement. [...] Non seulement je ne pense pas qu'il soit licite de faire prévaloir la notion de communauté de culture dans le cadre national, mais je crois qu'il appartient à l'historien de reconnaitre à quel point les différenciations culturelles font partie de la définition des rapports sociaux $^{55}$.

Cette posture trouvait assurément un écho favorable auprès d'une historiographie québécoise de plus en plus axée sur l'étude de la question sociale et plutôt hostile à une interprétation qui plaçait le "national», les événements politiques et l’identité au premier plan. Au métarécit national d’un Michel Brunet, Mandrou préférait assurément le métarécit libéral d'un Fernand Ouellet, dont la trame narrative, influencée par le libéralisme classique de Donald Creighton, envisageait prioritairement le progrès des valeurs libérales sur les plans économique, social et politique ${ }^{56}$. On peut aussi penser que cette inclination favorable à l'esprit du libéralisme convergeait avec une certaine tendance chez Mandrou à apprécier, dans ses propres travaux, le triomphe de l'Angleterre libérale et de sa "Glorieuse Révolution» sur l'Europe absolutiste ${ }^{57}$. N’allait-il pas lui-même jusqu'à accréditer la thèse de la "tolérance britannique» face à la population francophone du Canada pour expliquer une partie du phénomène de survivance nationale au Canada français?: «À longueur d'histoire, sur l'ensemble des deux siècles, de 1763 à nos jours, j'ai l'impression, écrit-il, que la politique des Britanniques puis des Anglo-canadiens a été plus libérale et plus ouverte que ne le disent bien des Québécois habituellement $^{58}$.» Il y a là une reprise évidente de la posture relativiste sur les

55. Robert Mandrou, «Histoire sociale et histoire des mentalités», 49, 230 (janvier 1972) : 43-44.

56. Sur les «métarécits» de l'historiographie québécoise, on consultera avec profit la thèse de Mathieu Arsenault, L'historiographie des Rébellions de 1837-1838 au XX'e siècle : débats et rôle structurant dans la construction des grandes représentations de l'histoire du Québec, mémoire de maîtrise (histoire), Université du Québec à Trois-Rivières, 2013.

57. À ce propos, voir notamment Robert Mandrou, Louis XIV en son temps - 1661-1715 (Paris, Presses universitaires de France, 1973).

58. Robert Mandrou, "Une société pluraliste», dans Le Canada au seuil du siècle de l'abondance (Montréal, Éditions HMH, 1969), 221. 
conséquences de la Conquête, posture longtemps privilégiée, s'il en est, par la tradition historiographique de l'Université Laval.

Il faut retenir d'autres traits qui, dans la continuité de la précédente démonstration, nous permettent de cerner la parenté idéologique entre Mandrou et les milieux scientifiques de Laval et d'Ottawa. Au risque de généraliser, il nous semble que c'était aussi autour de l'optique proprement "canadianiste» que ces savants communiaient. Le parcours des professeurs de l'Université Laval, en sociologie notamment, témoigne en effet d'une intégration plus marquée dans les réseaux universitaires canadiens. Selon Jean-Philippe Warren et Yves Gingras, cette intégration procéderait d'une même conviction selon laquelle les grandes institutions mises en place durant et après la guerre comme l'Office national du film, Radio-Canada et le Conseil des Arts "pouvaient servir à unir un pays divisé et que, au premier chef, la science, neutre et détachée, allait devenir le langage naturel des conversations nationales et permettre la "fusion des cultures" ${ }^{\prime 9}$ ". En histoire, cette conviction s'était au mieux exprimée à travers l'entreprise du Dictionnaire biographique du Canada, fruit d'une initiative conjointe entre professeurs d'histoire de l'Université Laval et de l'Université de Toronto au début des années $1960^{60}$. Elle avait aussi animé l'esprit des fondateurs de la revue Histoire Sociale/Social History précédemment évoquée.

Pour peu qu'il se soit prononcé sur les enjeux relatifs à la «question nationale» québécoise, c'est dans une perspective pour le moins résolument canadienne - celle de «l'État multinational ${ }^{61} »$, plus particulièrement - que Mandrou situait son questionnement sur les problèmes et défis posés aux hommes d'État canadiens. Dans une communication prononcée à l'occasion d'un colloque tenu au Centre culturel international de Cerisy-la-salle, en juillet 1968, Mandrou s'attachait à critiquer la thèse de la "classe ethnique» développée par le sociologue Marcel Rioux, au motif que cette dernière ne correspondait pas exactement à l'organisation sociale canadienne, dont les structures de pouvoir faisaient état d'un establishment francophone en expansion ${ }^{62}$. Autrement dit, la dualité nationale cana-

59. Jean-Philippe Warren et Yves Gingras, «Cinquante ans de recherches et de débats: fondation et évolution de Recherches sociographiques (1960-2010)", Recherches sociographiques, 52, 1 (2011): 125.

60. Voir André Vachon, "Le Dictionnaire biographique du Canada. Dictionary of Canadian Biography ", La Revue de l'Université Laval, 20, 6 (février 1966): 528-533.

61. R. Mandrou, «Propos de bienvenue» dans Le Canada au seuil du siècle de l'abondance, op. cit., 13.

62. Pour justifier son argumentation, Mandrou s'appuyait sur les données de l'ouvrage de John Porter, The Vertical Mosaic: An Analysis of Social Class and Power in Canada, Toronto, University of Toronto Press, 1965. 
dienne ne se présentait pas dans les paramètres exclusifs d'une situation de domination des francophones par les anglophones. Raison pour laquelle il fallait, à ses yeux, revenir à l'hypothèse du "dérivatif ethnique " selon laquelle «l'opposition entre les deux peuples fondateurs serait entretenue ou ravivée comme un dérivatif à des oppositions dont je dirais volontiers qu'elles sont plus fondamentales et sans doute plus menaçantes pour l'ordre social».

Cette analyse, que d'autres, comme Ouellet, avaient défendue au cours des années 1960, était rehaussée d'une conclusion à saveur résolument trudeauiste sur la complémentarité des cultures canadiennes et sa valeur exemplaire aux yeux d'une Europe ravagée par les guerres:

Dépassant le conflit entre les deux peuples, par la coexistence pacifique devenue, non plus un vœu pieux, mais une réalité qui comporte l'acceptation réciproque des uns par les autres, le Canada pourrait affronter et résoudre de façon canadienne, et non pas américaine [celle du «melting pot»], la crise de civilisation dans laquelle nous sommes engagés tous, vous et nous, de ce côté-ci de l'Atlantique comme de l'autre, et qui est la crise majeure de cette seconde moitié du $\mathrm{XX}^{\mathrm{e}}$ siècle ${ }^{63}$.

\section{L'historiographie des mentalités}

La communauté d'attitudes, de valeurs et d'opinion qui unissait Mandrou aux historiens de Laval et, plus largement, au climat intellectuel du Québec des années 1960, trouve un autre élément d'explication, nous semble-t-il, dans sa propre sensibilité heuristique: celle des mentalités. Celle-ci peut être analysée conjointement avec la réception positive que connaissait, au même moment, le paradigme sociologique de l'École de Chicago, dont certaines des lignes de force convergeaient avec l'historiographie des mentalités. Comprenons-nous bien: il ne s'agit pas ici de se faire équivaloir les méthodes et la théorie de ces deux traditions de pensée, qui ne manquèrent pas d'ailleurs de susciter débats et désaccords entre sociologues et historiens ${ }^{64}$. Tout au plus, souhaitons-nous montrer en quoi leur réception québécoise est fonction de certaines analogies significatives, à partir desquelles pouvaient converger des engagements de même nature.

La perspective des mentalités privilégiée par les Annales s'inscrit dans la lignée du renouvellement de l'histoire voulu par Henri Berr et Lucien Febvre. Ses divers emplois et inflexions à travers le temps n'épuisent pas

63. R. Mandrou, «Une société pluraliste», loc. cit., 224.

64. À ce propos, voir A. Dubuc, «L'influence de l’École des Annales au Québec», loc. cit., 370. 
la portée de son projet théorique, dans lequel plusieurs situent l'unité et la cohérence interne de la grande école historique française depuis ses premières formulations jusqu' aux années $1970^{65}$. C'est d'ailleurs en partie grâce à la notion de "mentalité» que les Annales françaises connaitront leur plus importante phase de rayonnement à l'échelle internationale. Pour Mandrou, qui figure parmi ceux qui actualiseront la notion au cours des années 1960, l'«histoire des mentalités» se référait à une branche de l'histoire socioculturelle. Elle s'inscrivait dans le projet plus vaste d'une histoire totale qui ressortait aux domaines de l'affectif, des sentiments, des sensibilités, des passions et des psychologies collectives ${ }^{66}$.

Plus particulièrement, les mentalités recouvraient, chez lui, les notions de "vision du monde" et de "psychologie sociale» ou collective, mais restaient irréductibles à la psychanalyse telle que conçue par ses fondateurs, qui constituait plutôt un "moyen de connaissance de l'individu». La perspective temporelle y était également cruciale, les structures mentales devant s'étudier "dans le cadre d'une histoire longue où les mutations sont lentes ${ }^{67} »$. Présenté comme un rempart contre les anachronismes, ce courant historiographique proposait aussi de restituer au mental collectif sa relative autonomie face aux déterminations plus pesantes du socio-économique et de la vie matérielle ${ }^{68}$. Souvenirs enfouis et sédimentés dans le pergélisol des cultures, expression authentique des inconscients collectifs, les mentalités désignaient aussi, sous la plume de Mandrou, ces "formes de résistances», en retard sur l'évolution rapide des modes de vie matérielle que composent les mutations industrielles, scientifiques et technologiques ${ }^{69}$. Ainsi se justifie la remarque de Philippe Ariès, pour qui l'étude des mentalités en histoire traduisait nécessairement "un souci constant de mieux comprendre le passage à la modernité ${ }^{70}$ ».

Il va sans dire que ce discours sur les mentalités entrait en résonance avec les directions que prenait le champ intellectuel du Québec des années 1950 et 1960, et le cadre dans lequel évoluaient les tendances nouvelles en sciences sociales, fortement influencées par l'anthropologie culturelle et le paradigme du "continuum rural-urbain» hérité des théories de la

65. Voir à ce sujet Nicolas Righi, «L'héritage du fondateur? L'histoire des mentalités dans l’École des "Annales" ", Le Philosophoire, 1, 19 (2003): 155-174.

66. Robert Mandrou, «L'histoire des mentalités», Encyclopedia Universalis, 8 (1970): 436.

67. R. Mandrou, «Histoire sociale et histoire des mentalités», loc. cit., 43.

68. Philippe Joutard, «L'Introduction à la France moderne et l’histoire des mentalités», dans R. Mandrou, Introduction à la France moderne..., op. cit., 489-490.

69. R. Mandrou, «L'histoire des Mentalités», loc. cit., 436-438.

70. Philippe Ariès, «L'histoire des mentalités», dans Jacques Le Goff, dir., La nouvelle histoire (Bruxelles, Éditions Complexe, 2006), 177. 
modernisation américaines et de la tradition sociologique de Chicago. Maintes analyses ont démontré l'empreinte que ces courants ont laissée sur les sciences sociales au Canada français, comme en témoigne notamment l'extension sociohistorique que donneront plusieurs sociologues québécois à l'application de l'étiquette «société traditionnelle» ou encore "Folk-society»" ${ }^{71}$ Ces réemplois répondaient alors à une variété d'exigences d'ordre idéologique et épistémologique. Devant l'intransigeance, le conservatisme et le moralisme d'une partie de la cléricature canadiennefrançaise, alliée de l'État duplessiste, l'opposition entre la tradition des sociétés rurales, communautaires, religieuses et la modernité des sociétés industrielles surgissait comme une configuration théorique idéale pour formuler une critique de l'Ancien Régime et de l'ordre établi ${ }^{72}$.

Le recours à ces cadres conceptuels permettait aussi de pondérer la problématique du "retard collectif » canadien-français, envisagée sous l'angle des mutations de la conscience ou de l'évolution des structures sociales. Une telle lecture invitait à dissocier les systèmes de représentations, les manières de penser, de sentir et de faire (autrement dit, la "culture» dans son sens anthropologique) des modes d'encadrement économique, politique et familiaux de la collectivité ${ }^{73}$. Cette dissociation avait pour finalité stratégique de mettre en relief le «décalage» entre l'infrastructure de la société, en rapide mutation, et la culture jugée "traditionnelle». Selon Warren, "c'est en creux de ce "culturalisme" que se perpétue l'idée d'une réforme des mentalités ${ }^{74}$ » dans le Québec francophone du tournant des années 1960, réforme engagée principalement par l'intelligentsia libérale de la revue Cité libre mais aussi par les sociologues de la Faculté des sciences sociales de l’Université Laval.

L'historiographie pratiquée par Mandrou et la tradition sociologique de Chicago s'installaient dans un même chantier des mentalités, qui relevait alors de la composante culturelle et psychologique de l'action humaine et des inconscients collectifs. Leur "resignification" dans le contexte québécois participait d'une même volonté d'inscrire l'analyse sous le schéma général du passage de la tradition à la modernité. Faut-il rappeler, à cette enseigne, que la typologie folk/urban avait aussi nourri

71. Voir à ce sujet Claude Couture et Claude Denis, «La captation des rapports entre tradition et modernité dans la sociographie québécoise », Canada: Theoretical Discourse/Discours théorique (Montréal, Association d'études canadiennes, 1994): 105-131.

72. Ibid.

73. Voir notamment Guy Rocher, Le Québec en mutation (Montréal, Les Éditions Hurtubise HMH, 1973).

74. J.-P. Warren, L'engagement sociologique..., op. cit., 291. 
la perspective des analyses historiques, dont plusieurs allaient retenir la lecture évolutionniste de l'histoire et l'interprétation «culturaliste» de la situation du peuple canadien-français? Les références à la problématique des «mentalités» reviennent d'ailleurs souvent sous la plume de certains historiens québécois de l'époque, en particulier ceux que côtoie Mandrou à Laval, qui l'employèrent dès les années 1950 toujours dans la rétrospective des immobilismes et des déphasages de la société canadienne-française. Claude Galarneau ne cherchait-il pas, déjà en 1955, "comment comprendre notre mentalité de refoulés, de casaniers, de xénophobes ${ }^{75}$ ?». Fernand Ouellet ne donnait-il pas une explication culturelle - la «mentalité» d'Ancien Régime - pour expliquer l'inaptitude des Canadiens français à s'adapter au capitalisme et à surmonter leurs problèmes conjoncturels ${ }^{76}$ ? Jean Hamelin ne dissertait-il pas sur la mentalité mégalomane des marchands en Nouvelle-France pour expliquer l'absence d'une bourgeoisie canadienne-française riche, forte et soucieuse du bien commun ${ }^{77}$ ? Tous venaient, eux aussi, alimenter le paradigme de la société rétive, restée cloisonnée dans des formes collectives archaïques ${ }^{78}$.

Il va sans dire que l'emploi du terme "mentalité» sous la plume des historiens québécois d'alors n'engageait pas forcément la même acception que lui avaient donnée Mandrou et l'historiographie française ${ }^{79}$. Voyons-y une conséquence directe de la labilité même du concept, dont le succès (tout comme les critiques d'ailleurs ${ }^{80}$ ) tient en partie à son étonnante capacité à agréger une pluralité d'objets dans les contextes les plus diversifiés $^{81}$. Cela dit, la présence marquée au Canada français d'un historien

75. Claude Galarneau, «Jeunesse de Clio ou la recherche en histoire», Revue d'histoire de l'Amérique française, 9, 1 (juin 1955), 9. (Les italiques sont de nous). Pour une application mieux circonscrite du concept, voir Claude Galarneau, "Toile de fond: histoire de la mentalité et des idées", dans P. de Grandpré, dir., Histoire de la littérature française du Québec. Tome 1 (Montréal, Beauchemin, 1967), 34-42.

76. F. Ouellet, Histoire économique et sociale du Québec..., op. cit.

77. J. Hamelin, Économie et société en Nouvelle-France, op. cit., 137.

78. Notons que cette perspective avait aussi été approfondie par des historiens comme Ouellet et Hamelin à la lecture des travaux d'Ernest Labrousse, qui faisaient alors autorité dans le domaine des courbes de prix. Le modèle labroussien de l'histoire économique et sociale postulait une hiérarchisation des trois instances de l'économique, du social et du mental, avec ses retards emboités (sur l'économique retarde le social et sur le social retarde le mental). Sur l'influence particulière de Labrousse chez les historiens lavallois, voir R. Rudin, Faire de l'histoire au Québec, op. cit., 161.

79. À ce propos, Nicole Gagnon souligne par exemple que Fernand Ouellet fait équivaloir la notion de "mentalités » à celle de "perception" plutôt qu’à celle de "représentation ", un glissement qui renverrait, selon elle, "à une conception assez primaire de l'idéologie " (Nicole Gagnon, "Compte rendu " [Le Bas-Canada, 1791-1840. Changements structuraux et crise], Recherches sociographiques, 19, 3 [1978], 409).

80. Geoffrey E. R. Lloyd, Pour en finir avec les mentalités (Paris, La Découverte/Sciences humaines et sociales, 1996).

81. François Dosse, «Histoire des mentalités», dans Christian Delacroix, François Dosse et Patrick Garcia, dir., Historiographies : concepts et débats, Tome 1 (Paris, Gallimard, 2010), 223. 
comme Mandrou, mais aussi celle d'Alphonse Dupront, tous deux associés à la sensibilité mentalitaire dans l'historiographie française, ne nous paraît pas anodine. Si ces derniers trouvèrent une terre d'accueil féconde au Québec, c'est assurément en raison du rapport d' "affinité élective» qu'ils purent développer avec l'orientation que prenait la pensée scientifique canadienne-française d'après-guerre.

\section{CONCLUSION}

Cette étude sur l'itinéraire de Robert Mandrou au Canada français portait l'ambition d'approfondir notre compréhension des circulations historiographiques entre la France et le Québec au tournant des années 1960. Plus exactement, il s'agissait d'examiner, dans une perspective d'histoire intellectuelle, les dynamiques d'adéquation et d'affinité entre d'une part, le parcours personnel et la pensée historienne de Mandrou, et d'autre part, l'horizon d'attente plus large des historiens québécois d'alors, plus particulièrement ceux des universités Laval et d'Ottawa. Nous avons vu que ce rapport était tributaire d'un ensemble de variables se rapportant à la réception particulière du paradigme annaliste au Canada francophone et à la spécificité des milieux de sociabilité fréquentés.

À tout prendre, il semble, au final, que ce soit davantage le Québec qui soit allé à la rencontre de Mandrou que l'inverse. L'historien français aura été un interlocuteur que la société québécoise recherchait, sciemment, à une heure où l'institutionnalisation de la science historique s'élaborait en concomitance avec les visées modernisatrices et rationalistes d'une nouvelle élite savante.

Il est intéressant aussi de signaler le rapprochement qui existe entre la sensibilité historiographique mentalitaire de Mandrou et l'interprétation "culturaliste» de la situation du peuple canadien-français que privilégiaient depuis quelques années certains chercheurs en sciences sociales et en histoire à Laval. Ainsi, l'analyse de sa présence et de son influence au Québec nous en apprend peut-être plus sur l'évolution de la société québécoise que sur Mandrou lui-même. Aussi, elle nous invite à privilégier une compréhension assurément plus complexe et circonstanciée des phénomènes de transferts intellectuels et théoriques entre le Québec et l'Hexagone. Ces phénomènes laissent entrevoir des procédés d' «appropriations créatives» dont la pleine nature resterait à établir dans le cadre d'autres analyses plus approfondies eu égard par exemple à la resignification du concept de «mentalité» ou encore à l'utilisation du drapeau de l' "histoire sociale», dont il est à supposer qu'il a convoqué diverses interprétations. 
Dans un autre temps, l'itinéraire québécois de Mandrou ne va pas sans interroger l'esprit et la configuration précise d'un lieu : celui du département d'histoire de l'Université Laval des années 1960, dont l'historicisation des idées, des individus et des pratiques reste encore un projet en chantier. Le mouvement de convergence entre Mandrou et les historiens qui évoluent ou qui ont été formés dans ce milieu souligne certains aspects de la personnalité propre à ce département. Le rapport d'affinité, nous l'avons vu, s'élabore tant sur le plan épistémologique (autour de l'histoire sociale et de l'histoire culturelle des mentalités) que sur le plan idéologique (par la mise à distance d'une historiographie jugée trop spontanément nationaliste). Il en résulte une autre inflexion évidente où le recours plus fréquent à des maîtres européens au département d'histoire à Laval venait assurément combler l'absence d'une figure tutélaire équivalente à celle du père GeorgesHenri Lévesque à la Faculté des sciences sociales ou encore de l'abbé Groulx au département d'histoire de l'Université de Montréal.

Mandrou s'est éteint à Paris le 25 mars 1984, victime d'une maladie qui le traquait depuis quelques années et qui, au grand malheur de ses "fidèles» québécois, l'avait obligé à diminuer considérablement ses activités de recherche et à mettre un terme à ses voyages au Canada. Ce décès précoce ne l'empêcha pas d'influencer plusieurs cohortes d'étudiants en histoire et de laisser une marque profonde dans le développement plus large d'une problématique d'histoire sociale et culturelle davantage consciente de ses méthodes et de ses objectifs au Québec. Sa veuve, Christiane Mandrou, constate d'ailleurs que l'œuvre de son mari fut dans une large mesure plus appréciée et reconnue à l'étranger qu'en France. À ce titre, elle rappelle justement que c'est à l'ambassade du Canada à Paris qu'a eu lieu, en janvier 1986, l'unique cérémonie officielle en l'honneur de sa mémoire ${ }^{82}$. Si l'événement est hautement révélateur pour l'historiographie québécoise, il l'est tout autant, sinon peut-être davantage, pour la mémoire disciplinaire de la France, qui semble avoir relégué l'apport de Mandrou dans les marges de l'oubli. Aux vues de la récente floraison d'études à caractère biographique sur certains historiens français à l'œuvre puissante $^{83}$, il est à espérer que le récit de vie du dernier disciple et confident de Lucien Febvre pourra trouver toute l'attention qu'il mérite.

82. Christiane Mandrou, «Le 27 juin $1995 »$, Les Cahiers du Centre de Recherches Historiques [En ligne], http://ccrh.revues.org/ 2555 [consulté le 06 novembre 2014].

83. Pensons notamment à Charles Mercier, Autonomie, autonomies. René Rémond et la politique universitaire en France aux lendemains de Mai 68 (Paris, Presses de l'Université Paris-Sorbonne, 2015); Christophe Prochasson, François Furet: Les chemins de la mélancolie (Paris, Éditions Stock, 2013); François Dosse, Pierre Nora: homo historicus (Paris, Perrin, 2011). 